\title{
DESARROLLO DE UNA PLATAFORMA WEB SIG PARA EL MONITOREO DINÁMICO DE PASTIZALES EN MAGALLANES
}

\author{
DEVELOP OF GIS WEB PLATFORM FOR GRASSLAND \\ DYNAMIC MONITORING IN MAGALLANES
}

Sergio Opazo (In memoriam) ${ }^{1}$, Eduardo Garay ${ }^{1}$, René Muñoz ${ }^{1}$, G. López-Saldaña² Rolando Aguilar ${ }^{1} \&$ Sergio Radic ${ }^{1}$

\section{RESUMEN}

Se presenta el desarrollo de un Sistema de Información Geográfica (SIG) WEB dinámico, el cual proveerá a los productores de la región de Magallanes una herramienta potente y versátil que les permitirá saber cómo ha variado el estado de los pastizales de sus predios a través del tiempo y contrastarlo con su condición actual, información importante a la hora de tomar decisiones de manejo. Para saber cuál es el estado de los pastizales se desarrolló el producto denominado Índice de Condición del Pastizal (ICP), el cual, se generó a partir de datos satelitales, específicamente a través imágenes MODIS (Moderate Resolution Imaging Spectroradiometer) a bordo de los satélites TERRA y AQUA. El sistema también consideró la incorporación de productos estáticos derivados de imágenes de alta resolución espacial como Worldview-2, IKONOS-2 y Geoeye-1 y de la generación de un sistema de transferencia de datos climáticos en tiempo real desde estaciones instaladas en terreno hacia la plataforma WEB desarrollada. El resultado es una plataforma WEB de fácil acceso e interpretación que permite al productor obtener información con alta frecuencia de actualización que le brinda un insumo importante a la hora de tomar decisiones de manejo.

Palabras clave: teledetección, praderas, Patagonia, plataforma WEB.

\section{ABSTRACT}

This document shows the development of a WEB platform based on Geographic Information System (GIS). This platform will be a powerful and versatile tool for Magellanic farmers, which will enable to check changes in their pastures condition through the years and be compared with the actual

$1 \quad$ Universidad de Magallanes, Punta Arenas, Chile. sergio.radic@umag.cl

2 Forest Research Center Technical University of Lisbon. 
condition. That is important information to collaborate in management decisions. With the aim to know the pasture condition, there was created a product called Pasture Condition Index (PCI), made with satellite information, specifically from MODIS (Moderate Resolution Imaging Spectroradiometer) images on board of TERRA and AQUA satellites. This system, also include static products from space high resolution images as Worldview-2, IKONOS-2 and Geoeye-1, and the generation of a transfer system for climatic information at real time, from field stations to the WEB platform. The results are an easy access and interpretation WEB platform that enables farmers to acquire information with update high frequency, considering this as an important tool for the management decisions.

Key words: remote sensing, grasslands, Patagonia, WEB platform.

\section{INTRODUCCIÓN}

La región de Magallanes se encuentra ubicada en el extremo sur del territorio chileno, siendo la más extensa del país. La superficie de esta región es de $132.291,1 \mathrm{~km}^{2}$, lo que representa el $17,5 \%$ del total del territorio nacional. Según Cruz \& Lara (1987), en el área de uso agropecuario de la región, la erosión es de carácter grave, no sólo por su intensidad, sino también por el grado de avance que dicho proceso está abarcando en la región. Confirmando la idea anterior, CIREN (2010) señala que la erosión en la región abarca 3,76 millones de hectáreas. Los problemas de erosión afectan extensas áreas de praderas naturales reduciendo su calidad y productividad, esto determina que la composición de especies de plantas varíe desde especies de mayor valor forrajero, a malezas de inferior valor (tanto herbáceas como arbustos). El aumento de especies de menor valor forrajero, resulta finalmente en que la pradera no aporta un buen sustento para mantener una adecuada carga animal en relación a su histórico, y en algunos casos, no protege adecuadamente el suelo, llegando al extremo de encontrar áreas con suelo descubierto.

El desarrollo espacial en torno al monitoreo de la superficie de la tierra mediante satélites especializados en esta área, y el vertiginoso avance en la teledetección, permiten en la actualidad contar con productos derivados de sensores remotos que posibilitan el monitoreo de variables importantes a la hora de definir la condición de una pradera de manera espacial y con una alta frecuencia temporal. Este monitoreo requiere de una gran base de datos y costosas mediciones en campo si se realiza con métodos tradicionales. Productos como índices de vegetación y temperatura de la superficie han sido ampliamente utilizados en el campo de los recursos naturales (Gill et al. 2009; Romo et al. 2012). Específicamente la aplicación de esta tecnología en el monitoreo de pastizales ya ha sido abordada en países como USA, Australia, Argentina, Colombia, entre otros (Reeves et al. 2001; Kaurivi et al. 2003; Watson et al. 2007; Wardlow et al. 2008; Van Leeuwen et al. 2008), ratificando la potencialidad de los productos como un insumo importante en el manejo de praderas.

Por otro lado, el desarrollo del concepto de mapeo WEB, permite hoy en día contar con una herramienta que permite suministrar datos geoespaciales a través de internet, facilitando el acceso de los usuarios a los datos desde cualquier lugar (que tenga acceso a la red) y sin límites de horario.

Este articulo tiene como objetivo, el desarrollo de una plataforma WEB SIG para el monitoreo dinámico de pastizales en Magallanes, que permita estimar la condición en relación a su histórico y la evolución de las praderas. Generando una plataforma WEB SIG (conjunto de hardware, software y datos geográficos) (Burk \& Lim, 1997) dinámica, para proporcionar a los productores una herramienta que les permita saber la condición de los pastizales de su predio de manera rápida, sencilla y de fácil acceso.

\section{MATERIALES Y MÉTODO}

\section{Consideraciones metodológicas generales}

Este trabajo abarca el sector sudamericano de la región de Magallanes, que se localiza en la parte sudoccidental desde los $48^{\circ} 36^{\prime}$ a los $56^{\circ} 30^{\prime}$ de latitud sur y entre los meridianos $66^{\circ} 25^{\prime}$ y $75^{\circ} 40^{\prime}$ de longitud oeste. Dentro de esta zona, se 


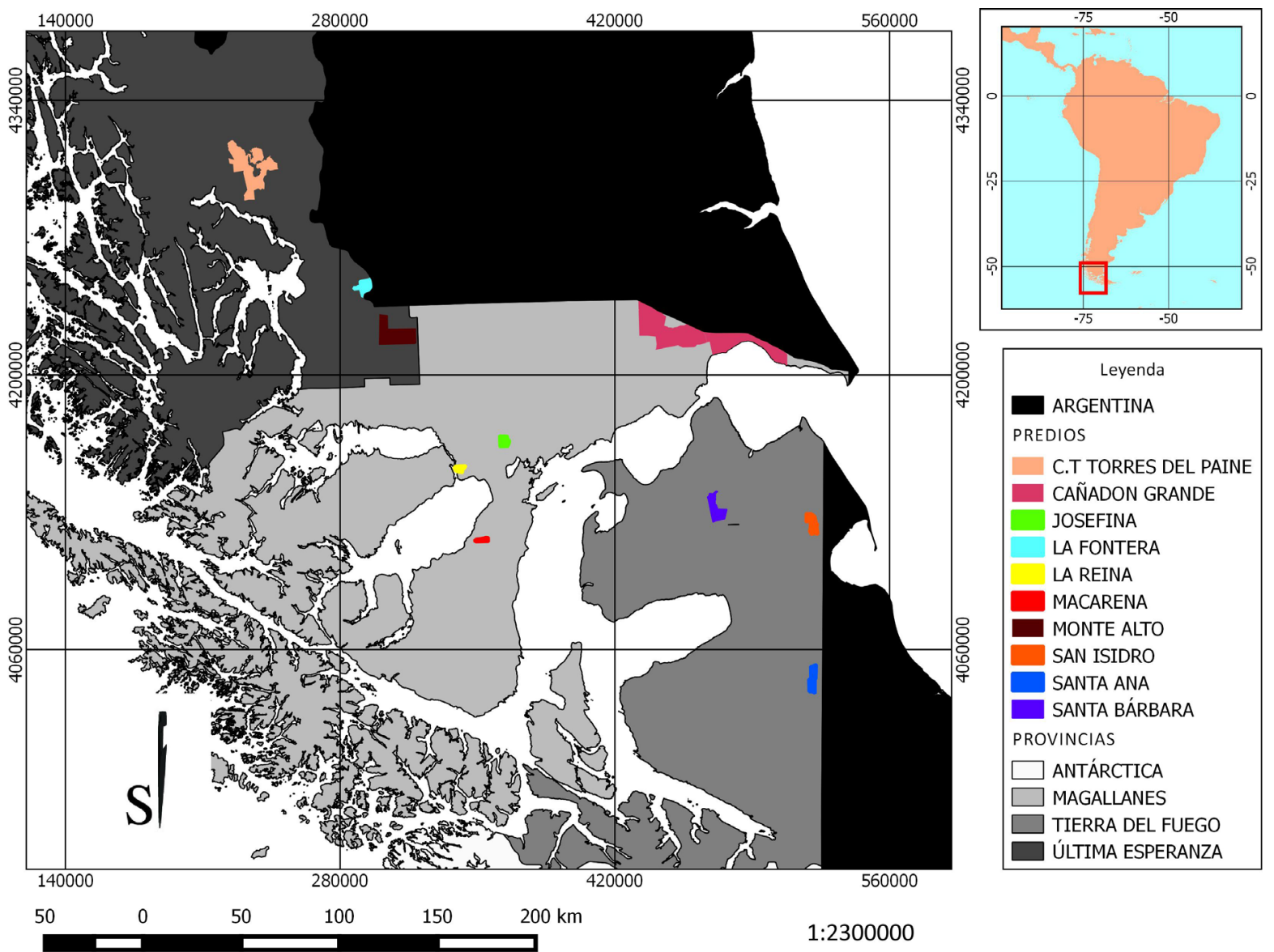

Fig. 1. Distribución geográfica de los sitios de estudio.

seleccionaron diez sitios para evaluar, considerando incluir a las distintas provincias de la región. De los sitios seleccionados, tres de estos se encuentran en la provincia de Tierra del Fuego, tres en la provincia de Última Esperanza y cuatro en la provincia de Magallanes. La distribución de los sitios en las diferentes provincias fue determinada en base al trabajo de los distritos agroclimáticos desarrollado por INIA (1982). La distribución geográfica de los sitios se presenta en la Fig. 1.

Para obtener la información sobre la condición de los pastizales se utilizaron datos obtenidos a partir de sensores remotos, específicamente imágenes del sensor MODIS a bordo del satélite TERRA (Running et al. 1994). Las imágenes MODIS son descargadas mediante una antena terrena y presentan una alta resolución temporal. El producto obtenido en primera instancia corresponde a un formato binario nativo nivel 0 , el cual es enviado diariamente a un servidor ubicado en la Universidad de Magallanes (UMAG). En el cual se procesan con el objetivo de obtener compuestos de 16 días para los siguientes productos: NDVI(Normalized Difference Vegetation Index), LST (Land Surface Temperature), Nieve y Evapotranspiración potencial. Dichos productos se almacenarán en una plataforma WEB a la cual tendrán acceso los productores.

También se adquirieron imágenes de alta resolución espacial (inferiores o iguales a $16 \mathrm{~m}^{2}$ ), dichas imágenes corresponden los satélites Worldview-2, IKONOS-2 y Geoeye-1 y fueron capturadas dentro del período de tiempo comprendido entre septiembre y abril (años 2009 a 2011). Estas últimas, fueron utilizadas para la generación de una clasificación de la vegetación de alto nivel de detalle espacial. Dicha clasificación no es utilizada como insumo en la generación 
del ICP, sin embargo permite al usuario conocer características cualitativas y cuantitativas de la vegetación en cada potrero y es una referencia importante a la hora de interpretar las variaciones que puedan existir en el ICP, las que pueden estar directamente relacionadas con el tipo de vegetación.

Para obtener datos meteorológicos de los predios se instalaron diez sensores Vaisala WXT520. Estos sensores son capaces de medir distintos parámetros atmosféricos entre los cuales podemos mencionar: temperatura $\left({ }^{\circ} \mathrm{C}\right)$, precipitación $(\mathrm{mm})$, humedad relativa $(\%)$, presión atmosférica $(\mathrm{hPa})$, velocidad $\left(\mathrm{km} \mathrm{h}^{-1}\right)$ y dirección de viento (grados). La información entregada por los sensores representa un complemento para la información generada a partir de las imágenes de alta resolución temporal, ya que permite explicar situaciones detectadas por los productos MODIS. Además, la información climática entregada por estas estaciones es una herramienta muy importante para mejorar la gestión predial (apoyo en la toma de decisiones).

Finalmente la información obtenida de las imágenes MODIS y de los sensores climáticos es presentada a los productores a través del SIG, por lo cual, pueden consultar por el estado de los pastizales de cada uno de los potreros de su predio en un período específico.

\section{Generación de productos \\ dinámicos de monitoreo}

\section{Creación de compuestos MODIS}

Los productos generados son una composición del promedio de todas las imágenes filtradas (libre de pixeles no deseados) cada 16 días, con esta cantidad de días se puede dividir el año en 23 periodos, por lo que finalmente obtendremos 23 imágenes por producto y por año. Se eligió la cantidad de 16 días para realizar los productos debido principalmente a que las condiciones climáticas de la región de estudio no permiten obtener una imagen con suficientes pixeles validos con un periodo menor y 16 días corresponde a un periodo suficiente para obtener imágenes sin cobertura de nubes en las zonas de estudio.

El procesamiento de los productos (NDVI,
ETP y LST) tiene una primera etapa que es común para todos, y después una etapa específica para cada uno, dependiendo el tipo de producto. Para este procesamiento se desarrolló un script en lenguaje de programación $\mathrm{C}$-Shell que se ejecuta automáticamente cada 16 días, las etapas de este script se describen a continuación.

\section{Preprocesamiento de las imágenes MODIS}

El primer paso consiste en filtrar las imágenes que cumplan con dos condiciones, que estén dentro del horario diurno y que cubran el sector de interés de acuerdo a las coordenadas geográficas. Si las imágenes pasan el primer filtro, se les aplican los scripts proporcionados por la aplicación SeaDAS, el primer script es el modis_L1A.csh el cual necesita como dato de entrada la imagen en formato binario nativo nivel 0 y como salida entrega un archivo de nivel L1A (MOD_PR01) del cual se obtiene su correspondiente archivo de geo-localización (GEO o MOD_PR03), utilizando el script modis_GEO. csh. Por último se utiliza el script modis_L1B.csh, el cual toma el archivo de nivel L1A y el archivo de geo-localización para generar el archivo de nivel L1B (MOD_PR02), con este último se puede crear directamente el producto LST. Sin embargo, para los productos NDVI y de Nieve se necesita obtener antes el producto de reflectancia (Reflect) MOD09. Para la creación del MOD09 se aplicó el script run_mod09.csh, posteriormente se procede a la extracción de las bandas necesarias para la creación de los productos NDVI y de nieve, las bandas extraídas son las siguientes: $250 \mathrm{~m}$ Surface Reflect Band 1, 250m Surface Reflect Band 2, $500 \mathrm{~m}$ Surface Reflect Band 4, 500m Surface Reflect Band 6 y $1 \mathrm{~km}$ Reflect Data State QA. Para extraer estas bandas se usó la aplicación MRT (Modis Reprojection Tool) Swath, la cual necesita de un archivo con los parámetros necesarios para su ejecución que definirán las bandas a extraer, la proyección, las coordenadas del área de interés, el formato de salida, entre otras opciones (Tabla 1).

\section{Filtrado de las Imágenes MODIS}

Un paso importante en la creación de los productos es el filtrado de las imágenes, esto se debe hacer debido a que las condiciones climáticas 
Tabla 1. Principales parámetros utilizados por la aplicación MRT Swath.

\begin{tabular}{lr}
\hline \multicolumn{1}{c}{ Parámetro } & \multicolumn{1}{c}{ Valor } \\
\hline INPUT_SDS_NAME & 250M_Surface_Reflect_Band_1; \\
& 250M_Surface_Reflect_Band_2; 500M_Surface_Reflect_ \\
Band_4; & \\
& 500M_Surface_Reflect_Band_6; \\
OUTPUT_SPACE_UPPER_LEFT_CORNER & 1km_Reflect_Data_State_QA \\
OUTPUT_SPACE_LOWER_RIGHT_CORNER & GEOTIFF_FMT \\
OUTPUT_FILE_FORMAT & UTM \\
OUTPUT_PROJECTION_NUMBER & -79.0502140509725 -45.65096371286147 \\
OUTPUT_PROJECTION_ZONE & $-62.5994768767874-56.27622870799083$ \\
OUTPUT_PIXEL_SIZE & 250 \\
\hline
\end{tabular}

de la región de estudio no permiten obtener en un solo día una imagen libre de pixeles no deseados como: nube, sombra de nube, entre otros, gracias a este filtrado es posible realizar la creación de los compuestos de promedios (de pixeles validos) cada 16 días.

Para poder realizar el filtrado se desarrolló un script en lenguaje Python, en el cual se utiliza la banda 1km_Reflect_Data_State_QA. Para filtrar la imagen, esta banda contiene distintos valores en cada píxel los cuales se encuentran en formato decimal. Si estos valores se transforman de decimal a binario (de 16 bits) podemos saber, a través de una tabla (Tabla 2), qué es lo que contiene ese píxel, esta tabla muestra los distintos valores que pueden tener los bits y que es lo que significan.

\section{Generación de productos}

dinámicos de NDVI y Nieve

Una vez que ya se han filtrado las imágenes se procede a crear el producto de NDVI (Huete et al. 1999) relacionado con la actividad

Tabla 2. Extracto de tabla de los valores de los píxeles.

\begin{tabular}{clcl}
\hline Nro. Bit & Parámetro & Combinación Bit & Estado del píxel \\
\hline $0-1$ & Estado de la nube & 00 & despejado \\
& & 01 & nuboso \\
& & 10 & variado \\
2 & Sombra de nube & 11 & no determinado, se asume limpio \\
& Tierra/Agua & 1 & si \\
& & 0 & no \\
& & 000 & océano poco profundo \\
& 001 & tierra \\
& 010 & costa de océano y costa de lago \\
& 011 & aguas continentales de poca profundidad \\
& 100 & corrientes de agua intermitentes \\
& 101 & aguas continentales profundas \\
& 110 & océano continental/moderado \\
& 111 & océano profundo
\end{tabular}


fotosintética (Chuvieco, 2002) y el producto de nieve, referido a la presencia o ausencia de dicha condición en un pixel. Ambos productos consideran las imágenes de los últimos 16 días, para esto se desarrolló un script en lenguaje Python, el cual promedia todas las imágenes filtradas (Bandas 1, 2, 4 y 6) para posteriormente realizar el cálculo del NDVI y de Nieve. formula:

El cálculo del NDVI está dado por la siguiente

$$
N D V I=\frac{\text { Infrarrojo Cercano }- \text { Rojo }}{\text { Infrarrojo Cercano }+ \text { Rojo }}
$$

Dónde:

Infrarrojo Cercano = Banda 1

Rojo $=$ Banda 2

Para la generación del producto de Nieve (Hall et al. 2000) se utilizaron tres criterios para identificar un pixel como nieve, estos deben cumplirse de manera simultánea.

\section{i) $\frac{\text { Banda } 4-\text { Banda } 6}{\text { Banda } 4+\text { Banda } 6}>0.4$}

ii) La reflectancia de la Banda 2 es mayor a 0.11 0.10

iii) La reflectancia de la Banda 4 es mayor a

Generación de productos dinámicos de LST y Evapotranspiración potencial (ETP)

Para la creación del producto LST (Ehrlich \& Lambin, 1996) se utilizó el script MODLST_ SPA el cual necesita como dato de entrada la imagen MODIS en formato L1B y el archivo GEO, como salida entrega el producto LST en grados Kelvin escalado por 10, posteriormente se transforman los valores a grados Celsius. Una vez que se tiene cada imagen en grados Celsius, se procede al filtrado de los pixeles para posteriormente crear el producto final con un script en lenguaje Python similar al ocupado para el NDVI.

El producto de ETP se calculó usando el producto de temperatura de superficie (LST) y la radiación extraterrestre ( $\mathrm{Ra})$, para esto se utilizó la siguiente fórmula (Radic et al. 2012):
$\mathrm{ETP}=\left(1-e^{-0.03 *(L S T+20)}\right) *\left((L S T+20)^{0.5} * R a * 0.408\right.$

Generación del Índice de

Condición del Pastizal (ICP)

El ICP es un indicador que tiene como función comparar la condición actual del pastizal (entregada por sensores remotos), en relación a su condición histórica, para un mismo período de tiempo. Este índice, es generado cada 16 días y permite a los usuarios emitir un juicio sobre el estado actual de un pastizal, contribuyendo en la toma de decisiones para un uso eficiente y sustentable de los recursos. El ICP está compuesto por los productos dinámicos NDVI y ETP. Sin embargo, con la finalidad de determinar variaciones en los niveles de NDVI en un mismo lugar, a lo largo del tiempo, se calculó la anomalía del NDVI (ANDVI). La fórmula que determina la ANDVI es la siguiente:

$$
A N D V I=\frac{\text { NDVI fecha } x-\text { NDVI promedio fecha } x}{\text { Desviación estandar fecha } x}
$$

Finalmente, el ICP está definido como:

$$
I C P=A N D V I-E T P
$$

Donde, ANDVI = Anomalía del NDVI, $\mathrm{ETP}=$ Evapotranspiración potencial.

Datos meteorológicos a partir de estaciones en terreno

Para la obtención de los datos meteorológicos se instaló una estación Vaisala WXT520 en cada predio. Se destaca que esta unidad en conjunto no posee ninguna parte móvil reduciendo al mínimo el mantenimiento de la misma (Sensor de viento ultrasónico y sensor de precipitación no invasivo). La estación es conectada a un sistema de adquisición de datos vía RS485 para adquirir los parámetros de temperatura, precipitación, humedad relativa, presión atmosférica, velocidad y dirección de viento, en periodos de 15 minutos. La información es almacenada localmente en una memoria micro SD y enviada en tiempo real desde un modem GPRS/GPS (GM862) hacia un servidor ubicado en la Universidad de Magallanes, utilizándose el protocolo de transferencia de datos UDP. Además, 

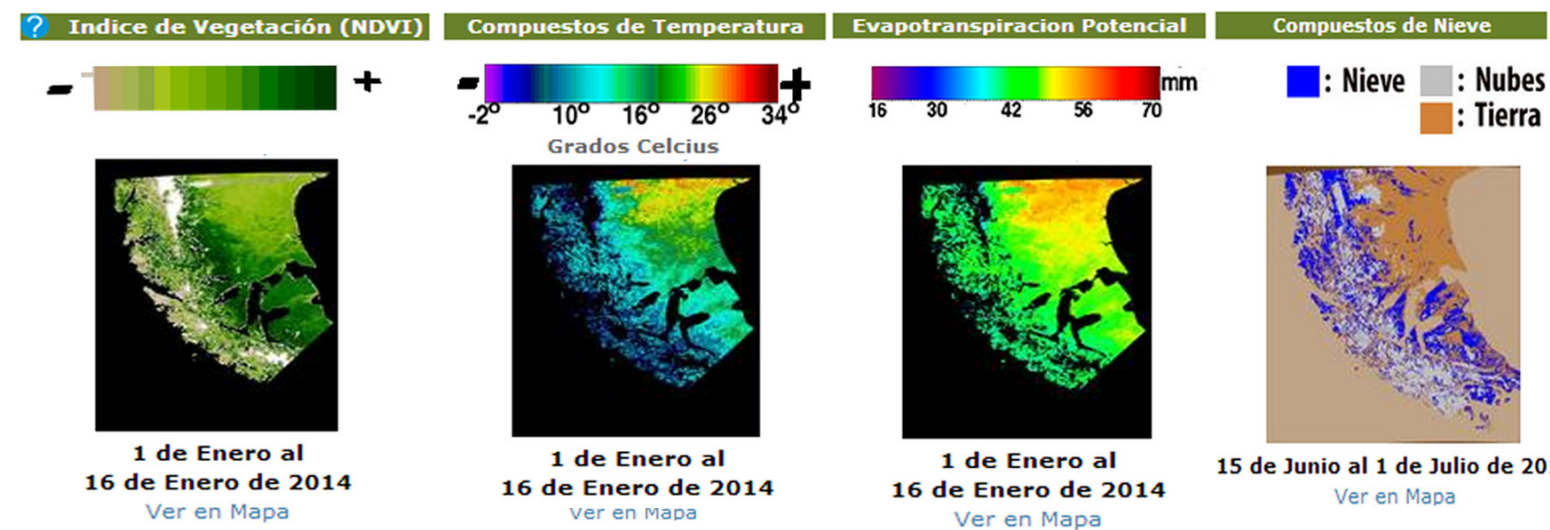

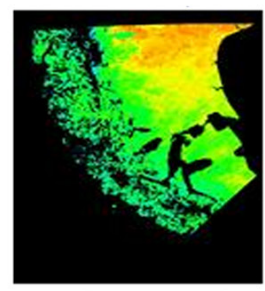

1 de Enero al

16 de Enero de 2014

Ver en Mapa

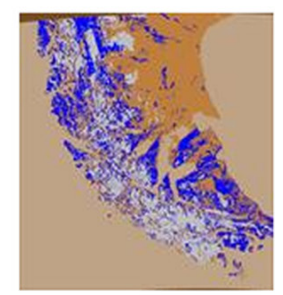

15 de Junio al 1 de Julio de 2012 Ver en Mapa

Fig. 2. Productos MODIS disponibles en la plataforma de acceso público (de izquierda a derecha: NDVI, LST, Evapotranspiración potencial y Nieve).

este modem es utilizado para adquirir la fecha, hora y ubicación GPS de la estación a través de la interpretación de secuencia de datos NEMEA.

En el servidor, se desarrolló un script en lenguaje PHP el cual se encarga de recibir los datos enviados por todas las estaciones a través del puerto 7110 UDP, este script está en constante ejecución a la espera de datos. Si un dato es recibido y se valida como correcto entonces se guarda en una base de datos MySQL para su posterior uso en la plataforma WEB SIG.

\section{WEB SIG}

La plataforma WEB se desarrolló con el objetivo de que los usuarios pudieran realizar consultas con respecto a los datos meteorológicos y a la información de los pastizales dentro de un potrero, en un período específico. Para lograr este objetivo utilizaron los siguientes componentes: para el servidor WEB se utilizó Apache, la interfaz se desarrolló con lenguajes HTML y JavaScript, para la base de datos se utilizó MySQL y para la lógica y conexión a la base de datos se utilizó PHP. Sistemas similares han sido desarrollados por Ferrán et al. (2013) y Gong et al. (2014).

Debido a que existe información exclusiva para los productores asociados, se crearon dos tipos de acceso al sistema, uno para el público general (acceso público) y otro para el acceso de los productores (acceso privado), proporcionando confidencialidad a los datos de los predios involucrados en el proyecto.

\section{RESULTADOS}

La plataforma generada cuenta con dos niveles de acceso (público y privado), los cuales presentan distinta información, opciones de visualización y de productos disponibles.

\section{Acceso Público}

Al ingresar al sistema se presentan las opciones para el público general, que incluyen: "Productos MODIS", "Estaciones climáticas", "Predios", "Series de Tiempo" y "Clima". Dentro de "Productos MODIS" se incluyen todos los productos generados por el sistema de monitoreo para que el usuario pueda buscarlos por mes y año. Esta búsqueda presenta un listado con los productos generados en la fecha seleccionada los cuales pueden ser visualizados en el mapa base de la plataforma (Fig. 2).

La sección de "Estaciones" permite al usuario ver en el mapa donde están ubicadas las 10 estaciones de monitoreo meteorológico, las cuales pueden aparecer con tres colores distintos: verde si está transmitiendo correctamente, amarillo si ha pasado más de un día sin transmitir datos y rojo si son más de dos días sin transmitir datos. Al hacer clic en una de ellas (estaciones) se desplegará una ventana con los últimos datos recibidos y un gráfico que muestra cómo se comportó la temperatura en los últimos 7 días. La sección "Predios" permite visualizar todos los límites prediales de la región. En la sección "Series de Tiempo" el usuario puede 


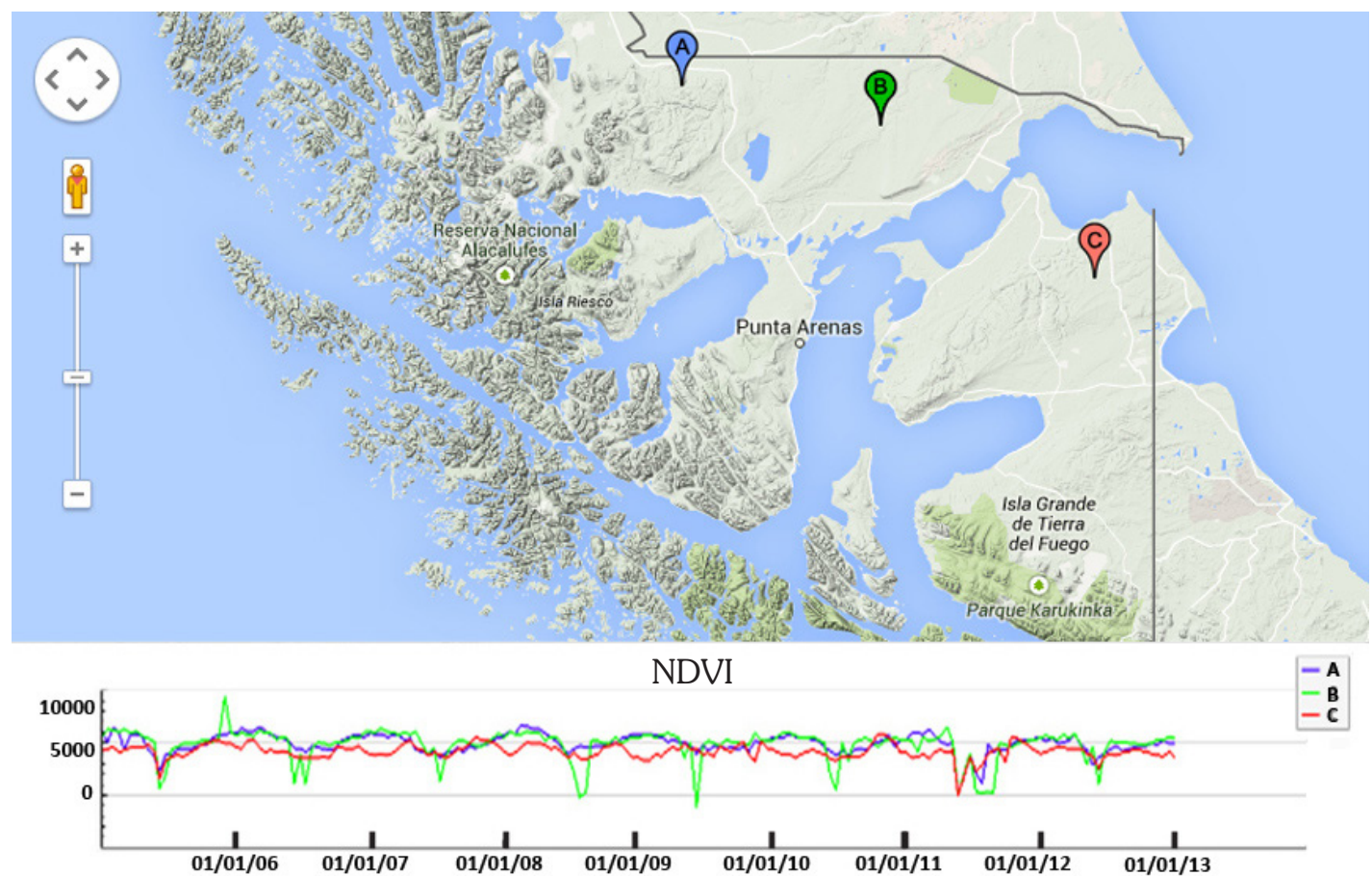

Fig. 3. Selección de tres puntos en el mapa y el respectivo gráfico comparativo para una serie de tiempo del producto NDVI.

seleccionar hasta tres puntos dentro del mapa, mostrando cómo se comportó el valor NDVI de los puntos seleccionados a través de los años en un gráfico que permite comparar los 3 puntos (Fig. 3). Por último dentro de la sección "Clima" se puede ver datos climatológicos generales de cada predio, es decir un resumen anual más un resumen del día actual, para saber información más detallada es necesario tener una cuenta de acceso de productor.

\section{Acceso Privado}

Los productores disponen de un acceso privado a la información detallada de sus predios, para esto se les asignó un nombre de usuario y una contraseña a cada uno. Cuando el productor ingresa al sistema se le muestra en el mapa el perímetro de los potreros de su predio junto con un símbolo en el centro de cada uno, este símbolo puede tener tres colores: rojo, naranjo o verde. El color rojo indica que el valor en el período actual del ICP de ese potrero es inferior a la desviación estándar obtenida del promedio de los valores históricos (desde el año 2005). El color naranjo indica que el ICP actual se encuentra entre el promedio histórico, y la desviación estándar bajo el promedio. Si bien determina una condición del pastizal desfavorable en relación a la condición promedio, ésta es una alerta para realizar seguimiento en el potrero, ya que las medidas a implementar se deberían tomar exclusivamente cuando el semáforo está de color rojo. Finalmente, el color verde indica que el ICP actual es superior al promedio de ICP de los años anteriores y que sus pastizales se encuentran en una condición superior al promedio histórico para ese mismo periodo (Fig. 4).

El productor también podrá desplegar una ventana emergente que contiene diversa información de ese potrero como: las comunidades vegetales, los valores actuales del monitoreo dinámico y un gráfico que compara los valores de un producto y período seleccionados a través del tiempo (Fig. 5).

Otros productos disponibles son: "Alta Resolución", "Series de Tiempo" y "Clima". En la opción "Alta Resolución" el productor puede visualizar las imágenes de alta resolución espacial para su predio, adquiridas en distintas combinaciones de bandas y algunos productos derivados de ellas como NDVI, comunidades 


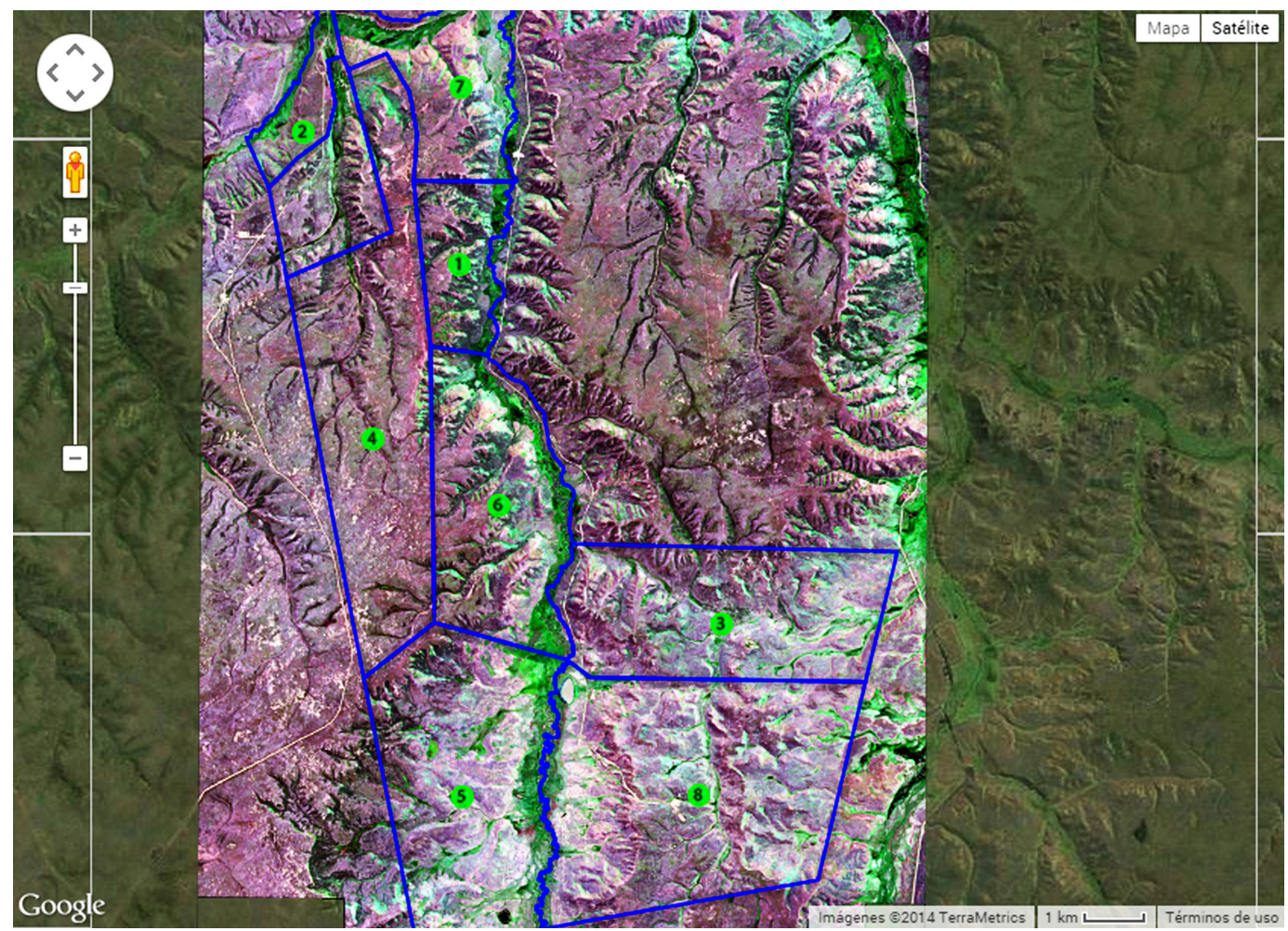

Fig. 4. Sistema de semáforos del índice de condición del pastizal para los potreros de un predio, desplegado sobre una imagen de alta resolución espacial (Geoeye-1) en combinación de bandas color real (3:2:1).

vegetales y valor ganadero, las que se pueden visualizar en el mapa con un gran nivel de detalle. En la sección "Series de Tiempo" el productor puede seleccionar un tipo de producto entre NDVI, ETP y LST, posteriormente, debe hacer clic en hasta tres puntos dentro de su predio, lo que generará un gráfico que muestra cómo se comportó el valor del producto elegido en los puntos seleccionados a través del tiempo. La sección "Clima" es similar a la del acceso público, sin embargo, el productor puede ver la información detallada por mes o día (Fig. 6) y adicionalmente generar gráficos para cada parámetro meteorológico presente en la base de datos de la plataforma WEB.

\footnotetext{
Comunidades Vegetales

Chilliotrichium diffusum: 154,34 ha ANDVI Actual: $0,71(0,00)$

Cuerpos de agua:

0,13 ha

243,56 ha

44,27 ha

1,61 ha

0,16 ha

6,14 ha

1,69 ha

451,95 ha

TOTAL:

Valor Ganadero:

5,81
}

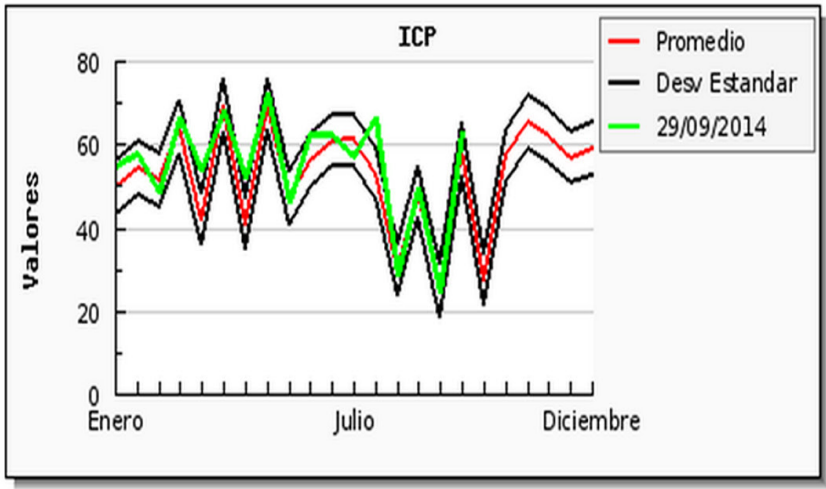

Fig. 5. Información detallada para un potrero determinado de un predio y gráfico del comportamiento histórico del índice de condición del pastizal en dicho potrero. 
Año 2014 - Estacion Puerto Natales

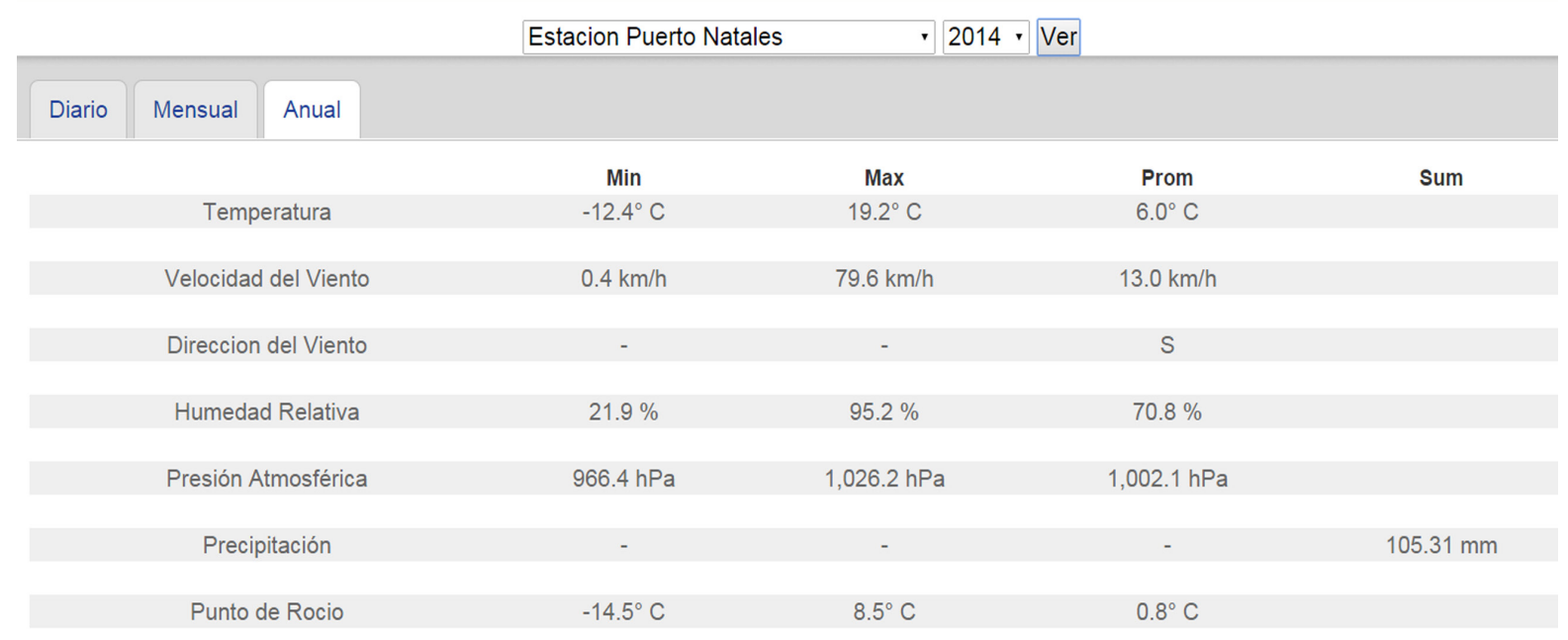

Fig. 6. Resumen de datos climáticos anuales para la estación climática ubicada en Puerto Natales.

\section{DISCUSIÓN}

Como se pudo observar en el desarrollo de este sistema, las imágenes MODIS fueron la principal fuente de información para conocer el estado de los pastizales. Esto fue posible debido a la gran versatilidad que tienen este tipo de imágenes, las cuales, no sólo son utilizadas para el monitoreo de la vegetación, además, su uso es muy variado y puede aplicarse a distintas áreas como: detección de áreas quemadas (Alonso-Benito et al. 2009), monitoreo de la deforestación (Moreno, 2013), cambios en composición de cobertura del suelo (García-Mora \& Mas, 2011) y monitoreo oceánico (Cerdeira-Estrada \& López-Saldaña, 2008; Cerdeira-Estrada \& López-Saldaña, 2011) entre otros usos. Otra ventaja del uso de las imágenes MODIS fue el hecho de que permitió generar un nuevo producto, el ICP.

El sistema de monitoreo de pastizales desarrollado en este trabajo, es el primero de su tipo en Chile, anteriormente han habido algunos trabajos donde se realizaron análisis con imágenes MODIS (Carvacho-Bart \& Sánchez-Martínez, 2010) pero la diferencia está en que el análisis fue hecho dentro de un periodo de tiempo específico en estos trabajos, o sea, los datos obtenidos eran estáticos, en cambio este sistema realiza un análisis nuevo de todos los sitios de estudio cada 16 días entregando valores promedios actualizados de
NDVI, Temperatura e ICP, esto ayuda a la toma de decisiones que pueda realizar el productor.

El filtrado de las imágenes fue un paso esencial para poder obtener una imagen libre de pixeles no deseados, este es un proceso común en trabajos con imágenes MODIS donde se desea obtener productos NDVI (Joseph \& Ross, 2006), temperatura de la superficie (Ke et al. 2011) o cualquier producto que necesite combinar varias imágenes en una sola.

Aunque el sistema fue desarrollado para monitorear diez sitios de estudio específicos, esto no impide que el mismo sistema se pueda implementar en otros predios de la región de Magallanes y en otras regiones o sectores del país. Como el sistema está desarrollado con software libre su implementación no requeriría de muchos cambios para adecuarlo a un nuevo sitio de estudio.

El sistema desarrollado es de gran ayuda para los productores de la región debido a la alta frecuencia de renovación de la información sobre los pastizales de sus predios, esto junto con los datos meteorológicos en tiempo real, permite que los usuarios puedan tomar mejores decisiones con respecto al manejo de sus pastizales. Esto es posible, debido a que el sistema funciona de forma autómata, es decir, el procesamiento de las imágenes y el llenado de la base de datos se hacen automáticamente cada 
16 días, con la información de los productos generados de las imágenes MODIS.

El hecho de trabajar con imágenes MODIS en su estado bruto, permitió tener un control total sobre toda la cadena de procesamiento, esto hizo posible la creación de productos personalizados como el ICP, así como también de los productos estándar, los cuales son accesibles desde el SIG. Debido a que se utilizaron mayoritariamente componentes Open Source para el desarrollo de este SIG, el costo con respecto a las herramientas utilizadas implicó que fuera muy bajo.

\section{AGRADECIMIENTOS}

El trabajo presentado en esta publicación fue parte de un proyecto Financiado por el Fondo de Fomento al Desarrollo Científico y Tecnológico (FONDEF D09I1036), el Servicio Aerofotométrico de la Fuerza Aérea de Chile (SAF), la Dirección General de Aeronáutica Civil (DGAC), el respaldo de diferentes empresas privadas del área agropecuaria de la región y la Universidad de Magallanes.

\section{LITERATURA CITADA}

Alonso-Benito, A., Hernández-Lealm, P., González-Calvo, A., Arbelo, M. y NúñezCasillas, M. \& Barreto, A. (2009). Cartografiado de áreas quemadas con imágenes MODIS y ASTER, Teledetección: Agua y desarrollo sostenible. XIII Congreso de la Asociación Española de Teledetección. Calatayud, 23-26 de septiembre de 2009. 277-280.

Burk, T. E. \& Lim, S. (1997). Internet tools Complementing Local GIS Processing, Proceedings of Integrating Spatial Information Technologies for Tomorrow, GIS'97, GIS World Inc., February 17-20, 1997. Vancouver, Canadá. 550-557.

Carvacho-Bart, L. \& Sánchez-Martínez, M. (2010). Comparación de índices de vegetación a partir de imágenes MODIS en la región del Libertador Bernardo O'Higgins, Chile, en el período 20012005. Ojeda, J., Pita, M.F. y Vallejo, I. (Eds.), Tecnologías de la Información
Geográfica: La Información Geográfica al servicio de los ciudadanos (pp. 728737). Secretariado de Publicaciones de la Universidad de Sevilla. Sevilla.

Centro de Información en Recursos Naturales (CIREN). (2010). Determinación de la erosión actual y potencial de los suelos de Chile. Región de Magallanes y Antártica Chilena. Síntesis de resultados. Centro de Información de Recursos Naturales. S. Flores (Ed). Santiago, Chile.

Cerdeira-Estrada, S. \& López-Saldaña, G. (2008). Automatic Processing of Near-Real Time Operational MODIS Ocean Products applied to Mexico Seas Monitoring, $5^{\text {th }}$ International Conference on Electrical Engineering, Computing Science and Automatic Control (CCE 2008) Art. $\mathrm{N}^{\circ}$. 4723456. pp. 545549.

Cerdeira-Estrada, S. \& López-Saldaña, G. (2011). A novel Satellite-based Ocean Monitoring System for Mexico, Ciencias Marinas, 37(2): 237-247.

Chuvieco, E. (2002). Teledetección ambiental. La observación de la tierra desde el espacio. Editorial ARIEL S.A Primera edición, Madrid, España.

Cruz, G. y Lara, A. (1987). Evaluación de la erosión en el área de uso agropecuario de la XII Región, Magallanes y de la Antártica Chilena. Instituto de Investigaciones Agropecuarias (INIA). Secretaria Regional Ministerial de Agricultura.

Ehrlich, D. \& Lambin, E. F. (1996). The surface temperature vegetation index space for land cover and land-cover change analysis. International, Journal of Remote Sensing, 17(3): 463-487.

Ferrán A., Bernabé, S., Rodríguez, P. G., \& Plaza, A. (2013). A Web-Based System for Classification of Remote Sensing Data. IEEE Journal of Selected Topics in Applied Earth Observations and Remote Sensing. 6(4): 1934-1948.

García-Mora, T. J. \& Mas, J.F. (2011). Evaluación de imágenes del sensor MODIS para la cartografía de la cobertura del suelo en una región altamente diversa de México. Boletín de la Sociedad Geológica Mexicana, 63(1): 
pp. 83-94.

Gill, T.K., Phinn, S.R., Armston, J.D., \& Pailthorpe, B.A. (2009). Estimating treecover change in Australia: challenges of using the MODIS vegetation index product. International Journal of Remote Sensing, 30, 1547-1565.

Gong, J., Wu, H., Jiang, W., Guo, W., Zhai, X. \& Yue, P. (2014). Geospatial Service Platform for Education and Research. The International Archives of the Photogrammetry, Remote Sensing and Spatial Information Sciences, Wuhan, China, Volume XL-6, 2014. ISPRS Technical Commission VI Symposium.

Hall, D.K., Riggs G.A. \& Salomonson, V.V. (2000). MODIS/Terra Snow Cover 5-Min L2 Swath 500m V004, December 2003 to March 2004. Boulder, CO, USA: National Snow and Ice Data Center. Digital media.

Huete, A., Justice, C. \& Leewen, W. (1999). MODIS vegetation index (MOD13). Algorithm theoretical basis document ATBD13, Digital Document: http://modis. gsfc.nasa.gov/data/atbd/atbd_mod13.pdf

Instituto Nacional de Investigaciones Agropecuarias (INIA). (1982). Plan de estudio Desarrollo Tecnológico Agropecuario. Vol. VI: Unidad de Trabajo $\mathrm{N}^{\circ} 3$. Distritos Agroclimáticos: Antecedentes. Estación Experimental Kampenaike. Punta Arenas. Chile.

Joseph, F. K. \& Ross, S. L. (2006). Regional Scale Land Cover Characterization Using MODISNDVI 250 m Multi-Temporal Imagery: A Phenology-Based Approach, GIScience \& Remote Sensing, 43(1) 1-23.

Kaurivi, J. Z., Huete, A. R., \& Didan, K. (2003). Multitemporal MODIS-EVI relationships with precipitation and temperature at the Santa Rita experimental range. USDA Forest Service Proceedings RMS-P-30, 121-124.

Ke, L., Wang, Z., Song, C \& Lu, Z. (2011), Reconstruction of MODIS LST Time Series and Comparison with Land Surface Temperature (T) among Observation Stations in the Northeast Qinghai-Tibet Plateau, Progress in Geography 30(7): 819-826.
Moreno, S. (2013). Cálculo de la tasa de deforestación anual en el periodo 20012011 en la provincia de Tambopatacon imágenes de NDVI (MOD13) de MODIS, mediante relación de ajuste de recta, Anais XVI Simpósio Brasileiro de Sensoriamento Remoto - SBSR, Foz do Iguaçu, PR, Brasil, 13 a 18 de abril de 2013, INPE.

Radic, S., Opazo, S., Muñoz, R. \& Mihovilovic, E. (2012). "Determinación de evapotranspiración potencial por medio de la teledetección". Proceedings Congreso Anual de la Sociedad Chilena de Producción Animal (SOCHIPA) (pp. 153154). XXXVII Reunión anual. Universidad de Concepción. Catillo, Chile.

Reeves, M. C., Winslow, J. C., \& Running, S. W. (2001). Mapping weekly rangeland vegetation productivity using MODIS algorithms. Journal of Range Management, 54(2): 207-207.

Romo Leon, J. R., Willem J.D. Van Leeuwen, G. M. \& Casady. (2012). Using MODIS-NDVI for the Modeling of Post-Wildfire Vegetation Response as a Function of Environmental Conditions and Pre-Fire Restoration Treatments. Remote Sensing. 4(3): 598621.

Running, S. W., Justice, C. O., Salomonson, V. V., Hall, D., Barker, J., Kaufman, Y. J., Strahler, A. H., Huete, A. R., Muller, J.P., Vanderbilt, V., Wan, Z. M., Teillet P. \& Carneggie, D. (1994). Terrestrial remote sensing science and algorithms planned for EOS/MODIS. International Journal of Remote Sensing, 15(17): 3587-3620.

Van Leeuwen, Willem J.D. \& Stuart E. Marsh. (2008). AVHRR and MODIS Vegetation Index Data for Rangeland Decision Support. Invited workshop speaker at the NIDIS Knowledge Assessment Workshop: Contributions of Satellite Remote Sensing to Drought Monitoring. February 6-7, 2008. David Skaggs Research Center, NOAA, Boulder, CO.

Wardlow, B. D., \& Egbert, S. L. (2008). Largearea crop mapping using time-series MODIS 250 m NDVI data: An assessment for the US Central Great Plains. Remote 
Sensing of Environment, 112(3), 10961116.

Watson, I. W., Novelly, P. E., \& Thomas, P. W. E. (2007). Monitoring changes in pastoral rangelands-the Western Australian Rangeland Monitoring System (WARMS). The Rangeland Journal, 29(2), 191-205. 
